\title{
Interaction of Forskolin with Voltage-Gated K+ Channels in PC12 Cells
}

\author{
Sarah S. Garber, ${ }^{a}$ Toshinori Hoshi, and Richard W. Aldrich \\ Department of Neurobiology, Stanford University School of Medicine, Stanford, California 94305
}

\begin{abstract}
Forskolin (FSK) directly blocks a distinct class of voltagedependent $\mathrm{K}^{+}$channels in pheochromocytoma cells. We have studied the biophysical mechanism of FSK action on these channels. The mean open duration decreased linearly with [FSK], indicating that a single molecule of FSK interacts with a single open $K^{+}$channel. FSK did not alter the voltage dependence of activation or the latency to first opening. Wholecell currents in the presence of FSK did not show a rising phase in tail currents, suggesting that FSK-bound channels can close. We used a kinetic scheme in which FSK binds preferentially to the open state of the channel to describe its interaction with the $K^{+}$channel. This scheme is analogous to the modulated receptor hypothesis used to describe the interaction of local anesthetics with voltage-dependent $\mathrm{Na}^{+}$ channels.
\end{abstract}

Forskolin (FSK), a ditcrpene isolated from the roots of Coleus forskohlii, is often used as an activator of adenylate cyclase. Because extracellular application of FSK has been shown to increase levels of intracellular cAMP, FSK is used to investigate cAMP-mediated modulatory effects in many different systems. It is now clear, however, that there are direct cAMP-independent effects of FSK on a variety of membrane proteins involved in ion or solute transporl, including voltage-dependent $\mathrm{K}^{+}$channels, nicotinic acetylcholine receptors, and the glucose transporter (Kashiwagi et al., 1983; McHugh and McGee, 1986; Grassi et al., 1987; Lavis et al., 1987; Wagoner and Pallotta, 1988; White, 1988).

In a previous article (Hoshi et al., 1988), we showed that FSK can accelerate the apparent inactivation kinetics and reduce the peak amplitude of delayed-rectifier-like whole-cell $\mathrm{K}$ currents in clonal pheochromocytoma (PC12) cells. The effects observed on the whole-cell currents were consistent with a direct cAMPindependent effect of FSK on the activity of a distinct class of voltage-dependent $\mathrm{K}^{+}$channels ( $\mathrm{Kz}$ channel). Similar cAMPindependent effects on voltage-dependent $\mathrm{K}$ currents have also been observed in other cell types (Coombs and Thompson, 1987; Watanabe and Gola, 1987; Krause et al., 1988; Harris, 1989). In this article, we studied the biophysical mechanism of the

Received Apr. 4, 1990; revised May 31, 1990; accepted June 6, 1990.

This work was supported by grant NS07158 to S.S.G., American Heart Association, California Affiliate Postdoctoral Fellowship to T.H., and grant NS23294 to R.W.A.

Correspondence should be addressed to Richard W. Aldrich, Department of Neurobiology, Fairchild D235, Stanford University School of Medicine, Stanford, CA 94305.

a Present address: Department of Physiology and Biophysics, University of Alabama at Birmingham, Birmingham, AL 35294.

Copyright (C) 1990 Society for Neuroscience $0270-6474 / 90 / 103361-08 \$ 03.00 / 0$ action of FSK on the $\mathrm{Kz}$ channel in PC12 cells. The results indicate that FSK preferentially interacts with the open state of the channel. In this regard, FSK block of the $\mathrm{Kz}$ channel resembles the effects of local anesthetics on voltage-dependent $\mathrm{Na}$ channels (Hille, 1977; Hondeghem and Katzung, 1977; Bean et al., 1983).

\section{Materials and Methods}

Cell culture. PC12 cells were obtained from the laboratory of E. M. Shooter, Stanford University, Stanford, CA. Cells were grown in Dulbecco's modified Eagle's medium (GIBCO Laboratories, Grand Island, NY) supplemented with $5 \%$ supplemented calf serum and $5 \%$ horse serum (Hyclone Labs, Logan, UT). Cells were maintained in plastic culture dishes and plated on borosilicate glass coverslips 1-14 $\mathrm{d}$ before use. All cells were grown in the absence of nerve growth factor.

Electrophysiology. Experiments were performed and the data analyzed essentially as described by Hoshi and Aldrich (1988a,b). All the singlechannel recordings were made in the outside-out configuration. Both whole-cell and single-channel data presented have been corrected for linear leak and capacitative currents. Arthimetic mean open times presented are not corrected for the high-frequency resolution limit of the recording system. Experiments were carried out at room temperature $\left(20-22^{\circ} \mathrm{C}\right)$

Solutions. The bath solution contained $140 \mathrm{~mm} \mathrm{NaCl}, 2.8 \mathrm{~mm} \mathrm{KCl}$, $2 \mathrm{mM} \mathrm{MgCl}_{2}, 1 \mathrm{mM} \mathrm{CaCl}_{2}$, and $10 \mathrm{~mm}$ HEPES, with the pH adjusted to 7.2 with $N$-methylglucamine. The pipette contained $140 \mathrm{mM} \mathrm{KCl}$, $11 \mathrm{~mm}$ EGTA, $2 \mathrm{~mm} \mathrm{MgCl}$, and $1 \mathrm{~mm} \mathrm{CaCl}\left(10 \mathrm{nM}\right.$ free $\left.\mathrm{Ca}^{2+}\right)$, with the $\mathrm{pH}$ adjusted to 7.2 with $N$-methylglucamine. Other solutions used are indicated in the figure legends. Working solutions of FSK were made up immediately before use from stock solutions of FSK (Sigma or Cal Biochem) in $95 \% \mathrm{ETOH}$ or $100 \%$ dimethyl sulfoxide stored at $-20^{\circ} \mathrm{C}$.

\section{Results}

Figure $1 A$ shows representative effects of FSK on the whole-cell voltage-dependent $\mathrm{K}$ currents in PC12 cells activated by depolarization. FSK reduced the peak amplitude and accelerated the apparent rate of inactivation of the current. The action of FSK was concentration dependent, causing graded changes in the peak current amplitude and apparent rate of inactivation with increasing concentrations of FSK (Hoshi et al., 1988). Normalized conductance-voltage curves (Fig. $1 B$ ) were not obviously altered in the presence of FSK, even though the peak amplitude of the whole-cell current was reduced. Although several different classes of voltage-dependent $\mathrm{K}$ channels exist in PC 12 cells, a single class of voltage-dependent $\mathrm{K}$ channels, called $\mathrm{Kz}$, is the dominant component of outward currents elicited by depolarization (Hoshi and Aldrich, 1988a,b). As shown in Figure $1 C$, and previously by Hoshi et al. (1988), the application of $120 \mu \mathrm{M}$ FSK to an outside-out patch reduced the activity of single $\mathrm{Kz}$ channels. The effects of FSK on single $\mathrm{Kz}$ channels are consistent with those of FSK on the whole-cell currents. 
Interactions of FSK with the open state of the channel

FSK markedly reduced the mean open duration of the $\mathrm{Kz}$ channel. In the data shown in Figure $2 A$, the mean open duration decreased from a control value of $18 \mathrm{msec}$ to $3.7 \mathrm{msec}$ in the presence of $120 \mu \mathrm{M}$ FSK. The distribution of open durations in the absence of FSK shows 2 exponential components: a minor fast component with a time constant of less than $1 \mathrm{msec}$, which comprises approximately $5-15 \%$ of the total openings, and the long major component with a time constant of $10-30 \mathrm{msec}$, depending on the voltage (Hoshi and Aldrich, 1988b). The control open-duration distribution shown in Figure $2 A$ was best fit by a sum of 2 exponential components with time constants of 1.2 and $23 \mathrm{msec}$. Their amplitudes were 0.15 and 0.85 , respectively. In the presence of FSK $(120 \mu \mathrm{M})$, the open-duration distributions were well described by a single exponential component with a time constant of $3.7 \mathrm{msec}$.

That FSK reduced the mean open duration indicates that FSK binds to a conductive conformation of the $\mathrm{Kz}$ channel. Therefore, we used the following simple mechanistic scheme as a starting point to further investigate the mechanism of FSK action:

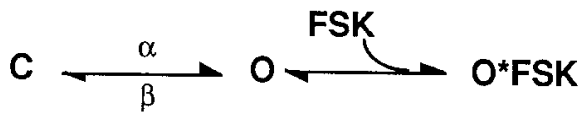

In this scheme, the channel moves from a nonconducting closed state, $C$, to an open and conducting state, $\mathrm{O}$, in response to a depolarization. We hypothesized that a single FSK molecule binds to the open state of the channel to make the channel nonconductive ( $\left.\mathrm{O}^{*} \mathrm{FSK}\right)$. In the following section, we test the predictions made by this hypothesis in order to develop a more detailed model.

For the sake of simplicity, all resting closed states of the $\mathrm{Kz}$ channel are included into a single-grouped closed state, C. Although $\mathrm{Kz}$ channels do undergo slow inactivation, closed states responsible for the inactivation are not included because the inactivation time course is much slower than the FSK action (Fig. 1). The open state responsible for the fastest of the 2 exponential components in the open-time histogram was also omitted because these brief openings only account for a small fraction of the total openings and charge transfer across the membrane (see Fig. 2A; Hoshi and Aldrich, 1988b).

According to the scheme described above, the mean open duration $\left(\tau_{\text {open(FSK) }}\right)$ of the channel in the presence of FSK is described as:

$$
\tau_{\text {open(FSK) }}=1 /\left(\beta+[\mathrm{FSK}]^{*} k_{\text {on }}\right) .
$$

The relationship between the mean open time of the channel and the rate at which FSK binds to the open state of the channel $\left(k_{\mathrm{on}}\right)$ can then be described as:

$$
1 / \tau_{\text {open(FSK) }}=[\mathrm{FSK}]^{*} k_{\text {on }}+1 / \tau_{\text {open(controll) }}
$$

and

$$
\tau_{\text {open(control) }}=1 / \beta .
$$

Therefore, the reciprocal of the mean open duration in the presence of FSK $\left(\tau_{\text {open(FSK) }}\right)$ should be linearly related to [FSK], if a single molecule of FSK binds to the open state of the channel. The slope of this relationship is $k_{\text {on }}$. The results plotted in Figure $2 B$ show that this relationship is linear, consistent with the hypothesis that a single molecule of FSK binds to the open, conducting state of the $\mathrm{Kz}$ channel. The binding rate of FSK, $k_{\text {on }}$, determined from the reciprocal of the mean open times, was typically $5 \times 10^{6}(\mathrm{sec}-\mathrm{M})^{-1}$.

\section{FSK binding does not depend on voltage}

Figure $3 A$ shows the voltage dependence of the mean open duration before and after applications of $120 \mu \mathrm{M}$ FSK. In the absence of FSK, the apparent mean open duration of the Kz channel was voltage dependent, increasing with greater depolarization (see also Hoshi and Aldrich, 1988b). The mean open durations in the presence of FSK were much less voltage dependent. This observation indicates that the interaction of FSK with the $\mathrm{Kz}$ channel is not markedly voltage dependent and that the rate-limiting step in the termination of the open cvent in the presence of FSK is the binding of FSK to the open state of the channel. The lack of perturbation of the conductance versus voltage curve in the presence of FSK (Fig. $1 B$ ) also supports this interpretation. The results thus indicate that the apparent voltage dependence of FSK action on the whole-cell currents was caused by the voltage dependence of the $\mathrm{Kz}$ channel activation.

The unbinding rate of FSK, $k_{\text {off }}$, may be calculated from the closed durations. The infrequent opening of $\mathrm{Kz}$ channels in the presence of FSK, however, prevented a reliable, direct determination of the unbinding rate of FSK, $k_{\text {off }}$, from the mean nonconductive time (see Fig. 1C). The dissociation rate of FSK is further discussed below.

\section{Interactions of FSK with the closed state of the channel}

The results presented above indicate that FSK binds, in a voltage-independent manner, to the open conformation of the $\mathrm{Kz}$ channel. According to Scheme 1, there should be no interaction of FSK with the closed states of the channel. We tested this hypothesis in the experiments described below.

\section{FSK does not affect $C$-to-O transitions}

We investigated the effect of FSK on the transition from closed to open conformations of the $\mathrm{Kz}$ channel by measuring the firstlatency distribution. The first-latency distributions are measures of the time it takes for a channel to move through a series of closed states to the first open, conducting conformation after the onset of the voltage step. The first-latency distributions obtained before and after the application of FSK did not differ markedly. The medians of the distributions before and after FSK application shown in Figure $3 B$ were 8.2 and $8.1 \mathrm{msec}$, respectively. This observation suggests that the steady-state binding of FSK to the closed states involved in the transitions from the resting closed states to the open state is not appreciable and supports the interpretation that FSK preferentially binds to the open conformation of the $\mathrm{Kz}$ channel.

The number of blank sweeps observed (i.e., number of voltage pulse epochs during which the channel failed to open) was not noticeably affected by FSK applications when the holding voltage was more negative than $-95 \mathrm{mV}$. However, when the holding voltage was more positive than $-95 \mathrm{mV}$, the channel failed to open more frequently in the presence of FSK.

\section{FSK-bound channels can close}

Scheme 1 requires that the channel must reopen before it closes so that FSK can unbind from the channel. According to this scheme, the tail currents observed following a voltage pulse to 
A

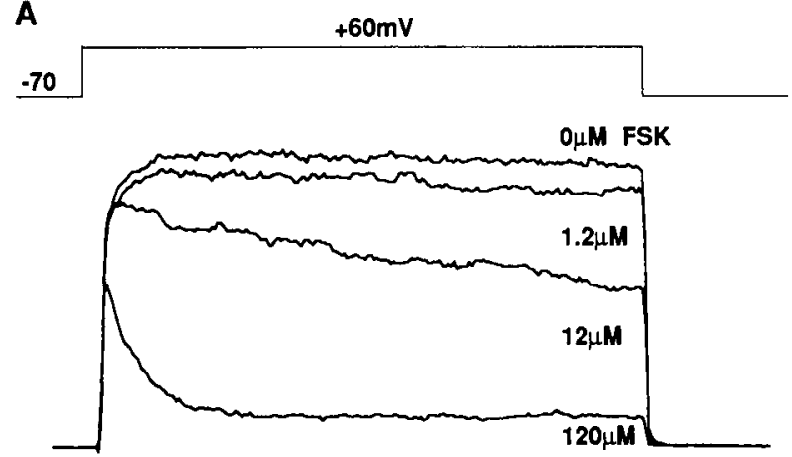

200pA

$30 \mathrm{~ms}$
B

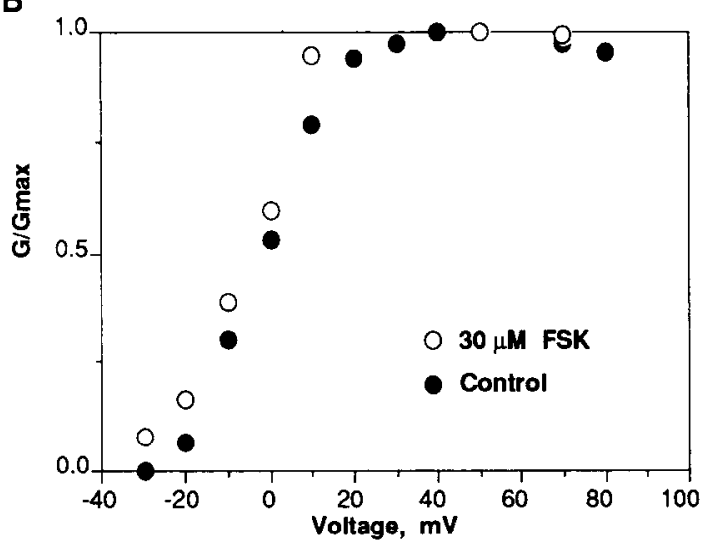

\section{C}

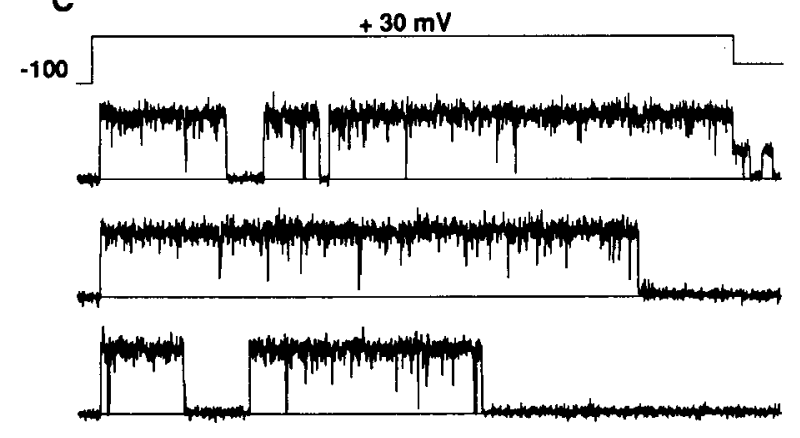

$+30 \mathrm{mV}$

$-100$
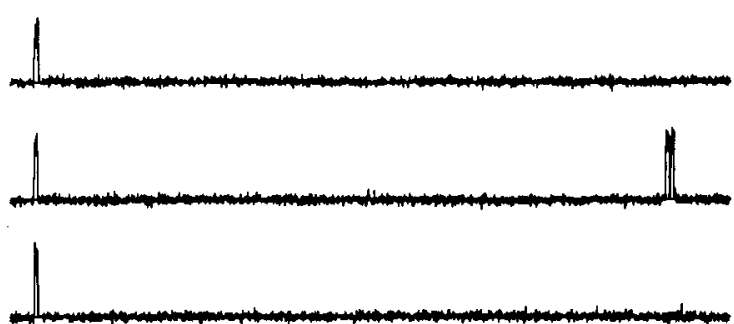

$2 \mathrm{pA}$

$120 \mathrm{~ms}$

Figure 1. Representative effects of FSK on Kz channel activity. $A$, Effects of bath applications of increasing concentrations of FSK on the wholecell outward currents. Concentrations and voltage protocols are indicated. The pulses were applied every $5 \mathrm{sec}$. All data shown were obtained from a single cell. $B$, Voltage dependence of the normalized conductance $\left(G / G_{\max }\right)$ was independent of [FSK]. Control data were obtained before the application of $30 \mu \mathrm{M}$ FSK to the bath. $G$ was calculated by measuring the amplitude of the peak outward current during the depolarizing voltage pulse and dividing by the driving force (rcversal voltage of the current was assumed to be $-70 \mathrm{mV}$ ). $C$, Representative openings of a Kz channel before and after $120-\mu \mathrm{M}$ FSK application to the extracellular side. The patch was depolarized to $+30 \mathrm{mV}$ from the holding voltage of $-100 \mathrm{mV}$, then to $-40 \mathrm{mV}$. The pulses were applied every $10 \mathrm{sec}$. The data were filtered at $1 \mathrm{kHz}$ and sampled at $3.3 \mathrm{kHz}$.
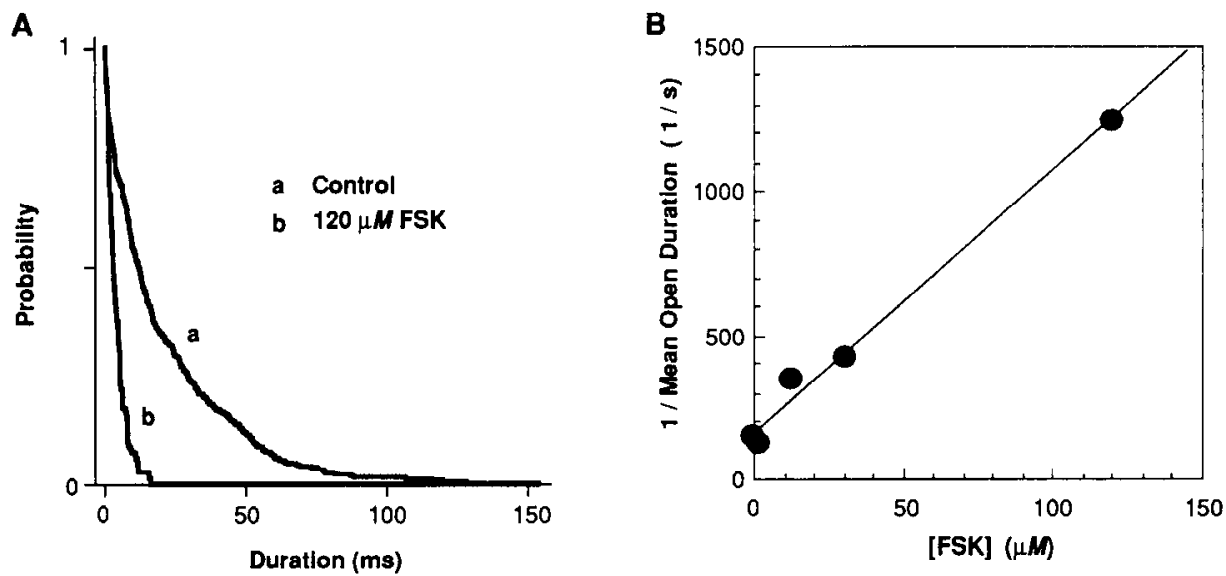

Figure 2. FSK decreases the mean open duration of the $\mathrm{Kz}$ channel. $A$, Open duration distributions of a single $\mathrm{Kz}$ channel before and after an application of $120 \mu \mathrm{M}$ FSK are shown. The data show the probabilities that a given open duration is greater than the durations indicated on the abscissa. The arithmetic mean open durations of the control and FSK openings were 18 and 3.7 msec, respectively. Control durations were best fit by a sum of 2 exponential components of 1.2 and $23 \mathrm{msec}$ (with amplitudes of 0.15 and 0.85 , respectively). Open durations in the presence of FSK were fit by a single exponential time constant of $3.7 \mathrm{msec}$. The openings were elicited at $+20 \mathrm{mV}$. The holding voltage was $-95 \mathrm{mV}$. The data were filtered at $1.2 \mathrm{kHz}$ and sampled at $10 \mathrm{kHz}$. $B$, The relationship between the reciprocal of the mean open duration and [FSK] is linear. The solid line was fit by linear regression analysis with a slope $\left(k_{\mathrm{on}}\right)$ of $4 \times 10^{6}(\mathrm{sec}-\mathrm{M})^{-1}$ and a y-intercept $(\beta)$ of $160 / \mathrm{sec}$. All the data points shown were obtained from a single experiment. The openings were obtained at $0 \mathrm{mV}$. The holding voltage was $-95 \mathrm{mV}$. The data were filtered at $1.2 \mathrm{kHz}$ and sampled at $10 \mathrm{kHz}$. Similar results were obtained from 3 other patches. 

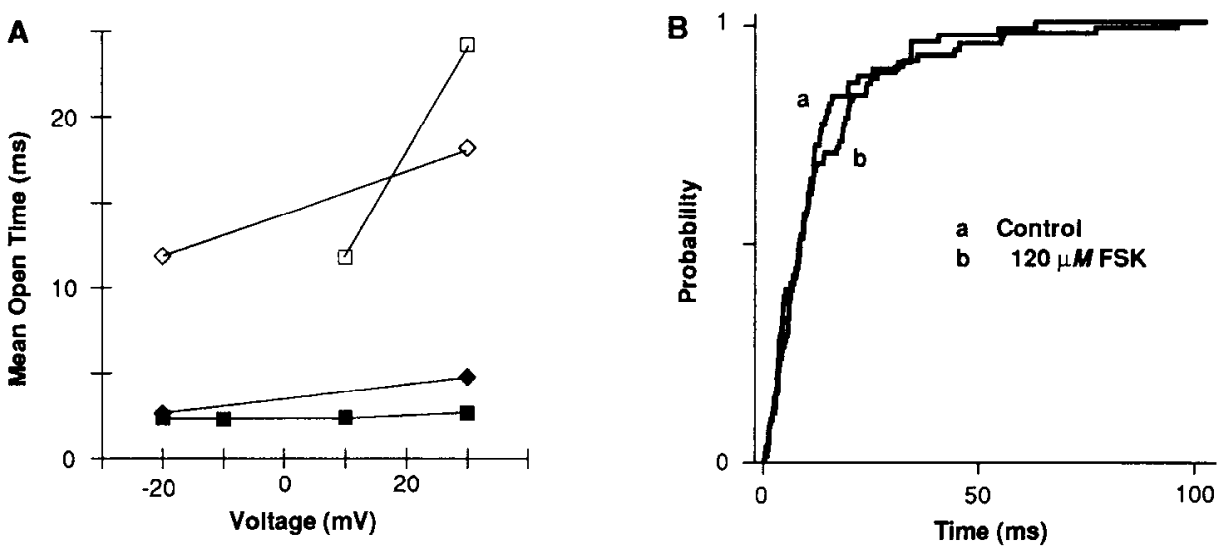

Figure 3. A, Mean open duration in the presence of FSK are not appreciably voltage dependent. Mean open duration of the control and FSKtreated $(120 \mu \mathrm{M})$ channel openings at different voltages are shown. The differently shaped symbols represent different experiments. Open symbols show the control arithmetic mean open times, and solid symbols show the mean open times after 120- $\mu \mathrm{M}$ FSK applications. The data were filtered at $0.8-1.2 \mathrm{kHz}$ and sampled at $10 \mathrm{kHz}$. B, First-latency distributions do not change markedly in the presence of $120 \mu \mathrm{M}$ FSK. First-latency distributions show the probabilities that the channels have opened by the times indicated on the abscissa after the voltage pulse onsets. Medians of the first-latency distributions before and after the FSK application were 8.2 and $8.1 \mathrm{msec}$, respectively. Only the depolarizing epochs that elicited at least 1 opening were included. The data were obtained at $-20 \mathrm{mV}$ before and after an application of $120 \mu \mathrm{M}$ FSK. The holding voltage was -95 $\mathrm{mV}$. The data were filtered at $1.2 \mathrm{kHz}$ and sampled at $10 \mathrm{kHz}$. The pulses were applied every 4 sec. This patch contained only 1 active channel. Medians of the first-latency distributions measured at other voltages $(-25$ to $+30 \mathrm{mV})$ were not affected by $120-\mu \mathrm{M}$ FSK applications.

a voltage positive enough to activate most of the $\mathrm{Kz}$ channels $(>5 \mathrm{mV}$ ) should have a rising phase reflecting the transition of the channels from the FSK-bound, nonconducting state to the FSK-free, open state on the way to the resting closed state(s). Tail currents (Fig. 4) were measured in a symmetrical $\mathrm{K}^{+}$solution to increase their amplitudes. In the control conditions, tail currents decayed slowly following a multiexponential time course. In the presence of FSK, tail currents were markedly reduced in amplitude, and no noticeable rising phase in the tail currents was observed.

The tail currents shown in Figure 4 werc followed for 300 msec following a hyperpolarizing pulse. After $300 \mathrm{msec}$ at a hyperpolarized voltage, at least $50 \%$ of the $\mathrm{Kz}$ channels should be free of FSK (see Fig. 5). In addition, for all experiments, current levels recovered with a repetition interval between command voltage pulses of $>3 \mathrm{sec}$. The absence of any rising phase of the tail current during this time suggests that $\mathrm{Kz}$ channels do not have to reopen for FSK to unbind from the channels. Contrary to the mechanism described in Scheme $1, \mathrm{Kz}$ channels must be able to close when FSK is bound, and FSK must be able to dissociate from the channel even when it is closed.

\section{Recovery from FSK block}

A 2-pulse protocol was used to estimate the rate at which FSK dissociates from the closed state of the channel. Using this protocol, the first depolarizing pulse would open channels and allow FSK to bind. The current amplitude during the second pulse reflects the fraction of the channels that had recovered from the FSK block during the interval between the 2 depolarizing pulses. This recovery represents the rate of unbinding of FSK from the closed channel, or $k_{\text {off, }}$, because there is little steadystate binding to the closed states. In the experiment shown in
Figure 4. Tail currents obtained in the presence of FSK did not show appreciable rising phases. Families of currents were recorded at the various voltages using the pulse protocol shown in the presence of $140 \mathrm{mM} \mathrm{K}^{+}$in the external solution. Application of $120 \mu \mathrm{M}$ FSK substantially decreased the amplitude of the tail currents. The bath solution contained $140 \mathrm{~mm} \mathrm{KCl}, 2 \mathrm{~mm}$ $\mathrm{MgCl}_{2}, 1 \mathrm{mM} \mathrm{CaCl}_{2}$, and $10 \mathrm{~mm}$ HEPES, with the $\mathrm{pH}$ adjusted to 7.2 with $\mathrm{N}$-methylglucamine. The pipette contained $140 \mathrm{~mm} \mathrm{KCl}, 11 \mathrm{~mm}$ EGTA, 2 $\mathrm{mM} \mathrm{MgCl}, 1 \mathrm{mM} \mathrm{CaCl}_{2}$, and $10 \mathrm{nM}$ free $\mathrm{Ca}^{2+}$, with the $\mathrm{pH}$ adjusted to 7.2 with $N$-methylglucamine.
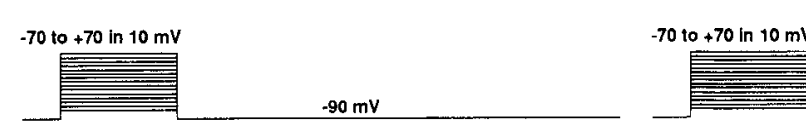
$.90 \mathrm{mV}$
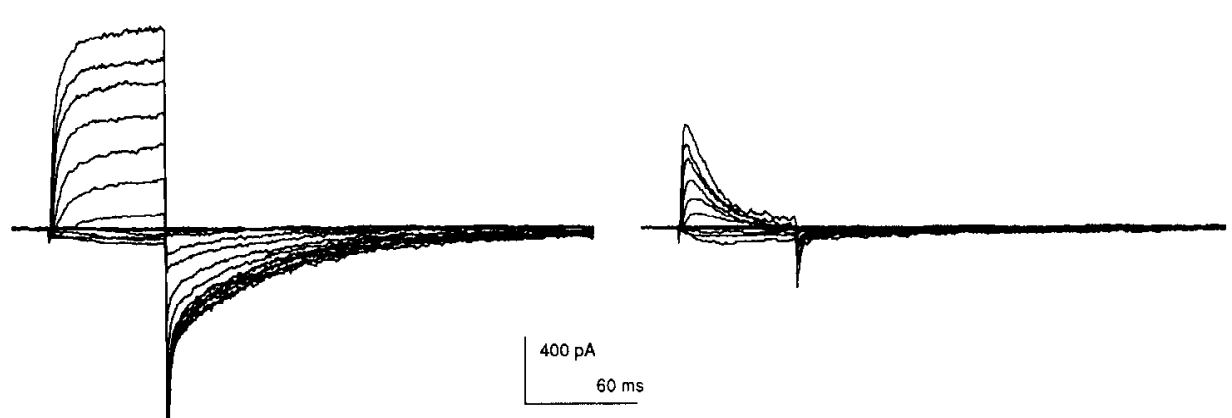
A

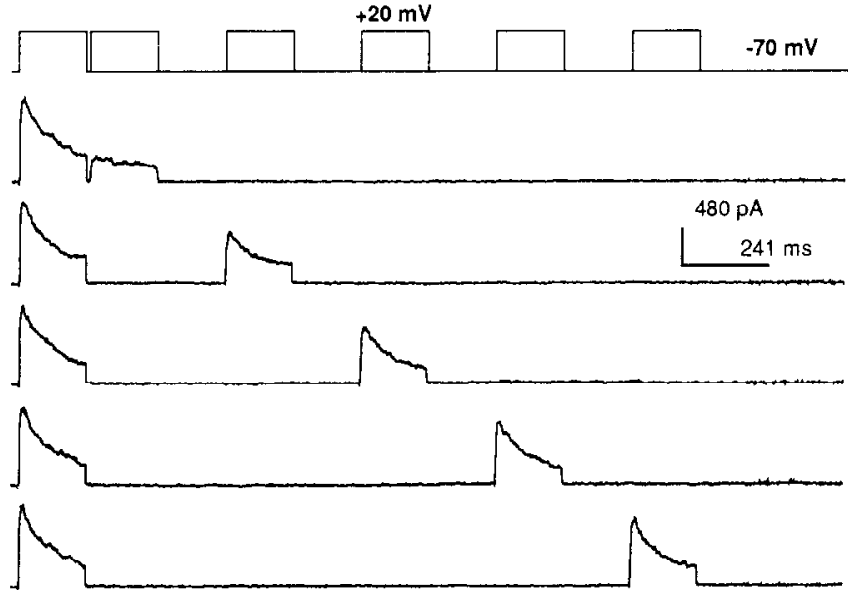$$
\mathbf{B}
$$ \\ $\mathbf{B}$}

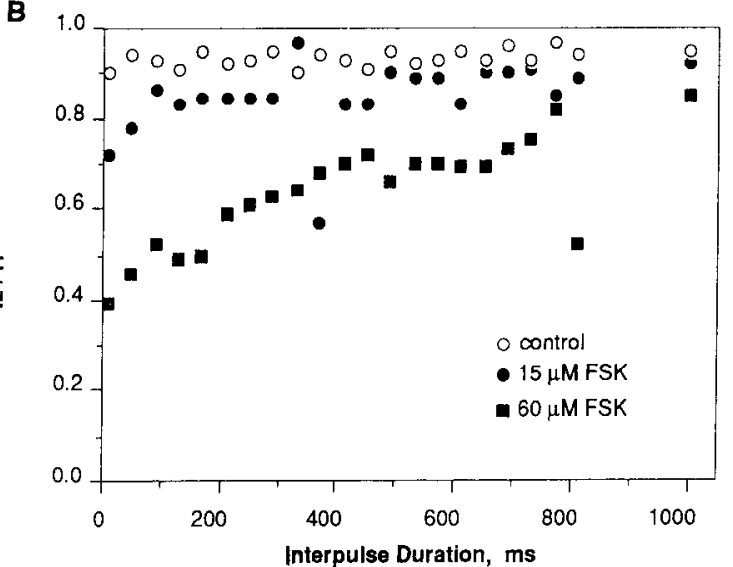

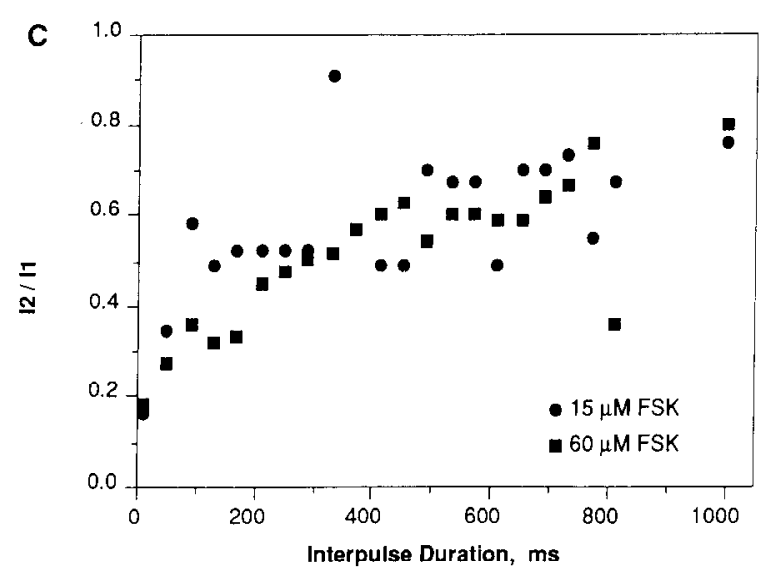

Figure 5. Recovery from FSK block. In the 2-pulse paradigm illustrated in $A$, the interpulse duration was varied from 10 to $1910 \mathrm{msec}$. The pulse protocol was applied every $5 \mathrm{sec} . B$. The ratio of the peak current in the second command pulse $\left(I_{2}\right)$ to the peak current during the first command pulse $\left(I_{l}\right)$ is plotted as a function of the interpulse duration. $C$. The rate of recovery from FSK block is not dependent on [FSK]. The ratio of $\mathbf{I}_{2} / \mathbf{I}_{1}$ from $B$ in the presence of 15 or $60 \mu \mathrm{M}$ FSK was normalized by extrapolating a y-intercept value, subtracting that value from the data points, and scaling the resulting value by the maximal value. Each point represents the average of $\geq 3$ experiments.

Figure $5 A$, the interpulse interval was varied from 10 to 1910 msec. The interpulse voltage was $-100 \mathrm{mV}$ so that there was a negligible activation of the channel during this interval. As the interval between the 2 depolarizing steps was increased, a greater amount of recovery was observed. However, though $50 \%$ of the recovery occurred within the first $\sim 300 \mathrm{msec}$ (Fig. $5 B$ ), complete recovery required more than $2 \mathrm{sec}$. Hence, the rate $\left(k_{\text {off }}\right)$ at which FSK leaves the $\mathrm{Kz}$ channel, at the voltages where the channel activation is negligible, is estimated to be about $4 /$ sec or less.

\section{Independence of recovery rate on [FSK]}

If FSK binds only to the open state of the channel, only the blocking rate of FSK will be dependent on [FSK]. Unblocking of the channel or the recovery from the FSK block should be independent of [FSK]. The rates of recovery of the whole-cell currents in the presenc eof 15 and $60 \mu \mathrm{M}$ FSK were compared using the 2-pulse protocol with variable interpulse interval described above. In the presence of $60 \mu \mathrm{M}$ FSK, a greater fraction of the current remained blocked for a given interpulse duration. However, the rates of recovery of the current, as measured by normalizing the curves shown in Figure $5 B$, were similar in 15 and $60 \mu \mathrm{M}$ FSK (Fig. 5C), suggesting that dissociation of FSK from the channel is independent of [FSK].

\section{Voltage dependence of recovery from FSK block}

Recovery of the $\mathrm{Kz}$ channel from the FSK block was dependent on the voltage. Greater hyperpolarization increased rates of recovery. Voltage dependence of recovery from FSK block was studied by varying the interpulse voltage in a 2-pulse protocol similar to that described above. Figure 6 shows that, in the presence of FSK, the peak current amplitude recovered faster with an interpulse voltage of $-100 \mathrm{mV}$ than one of $-70 \mathrm{mV}$. Therefore, the dissociation rate of FSK from the $\mathrm{Kz}$ channel becomes very large when the membrane voltage is set very negative.

\section{A modulated receptor mechanism of FSK action}

The results described above suggest that, though FSK binds preferentially to a conductive conformation of the channel, the channel is available to open again within a few seconds of repolarization. Also, with hyperpolarization, an FSK-bound channel can close without unbinding. Because FSK does not noticeably affect the first-latency distribution, FSK binding to the resting closed states is negligible at very negative voltages. The characteristics of this FSK block of the $\mathrm{Kz}$ channel resemble those of the blocking by local anesthetics of voltage-dependent Na channels (Hille, 1977). The interaction of FSK with the $\mathrm{Kz}_{z}$ 


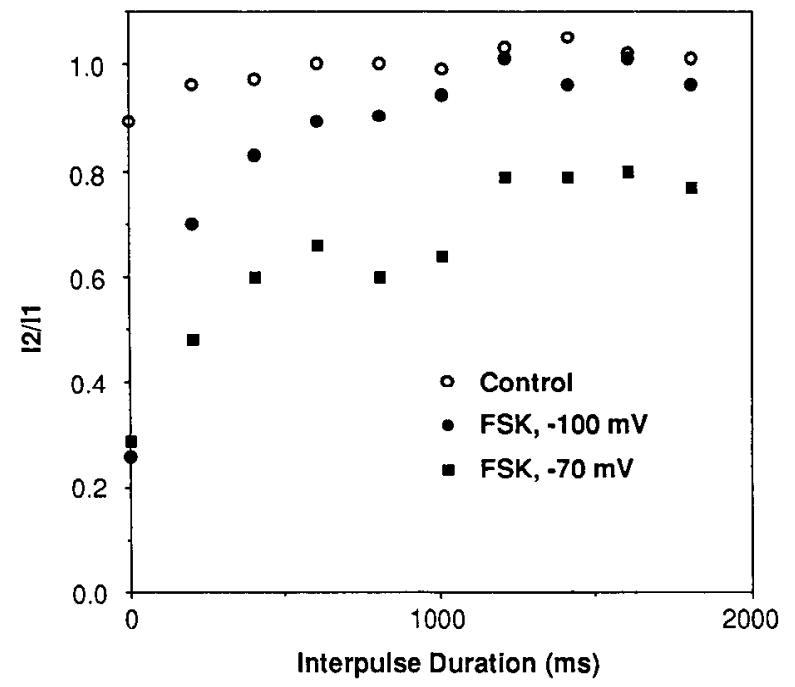

Figure 6. Recovery of FSK block is dependent on interpulse voltage. More negative interpulse voltages [ $-100 \mathrm{mV}$ (solid circles) vs $-70 \mathrm{mV}$ (solid squares)] increased the recovery rate at a constant [FSK] $(60 \mu \mathrm{M})$. The interpulse voltage of the control points (open circles) was $-70 \mathrm{mV}$. The pulse protocol used for these experiments was the same as that described in Figure 5A. Each point represents the average of $\geq 3$ experiments.

channel is more complex than described by Scheme 1, above. We have used a modulated-receptor-type scheme to describe the interaction of FSK and the Kz channel (Scheme 2):

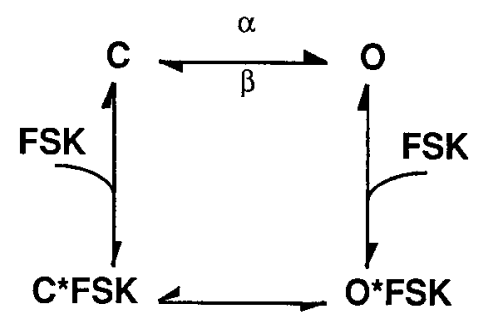

For the present discussion, we have ignored the inactivated state in the presence and absence of FSK, lumped the closed states, and ignored a fast open state as described above.

\section{Effect of FSK on whole-cell current}

The effect of FSK on the whole-cell current could be modeled using expcrimentally derived values for rate constants. The rates of the opening and closing transitions in the absence of FSK were empirically determined by fitting currents elicited in response to a pulse to $+50 \mathrm{mV}(\alpha=250 / \mathrm{sec}$ and $\beta=10 / \mathrm{sec}$, respectively). The binding rate of FSK to the open channel, experimentally determined to be on the order of $10 \% / \mathrm{sec}$, is fast compared to the unbinding rate of FSK from the open channel. Because of the absence of an FSK effect on first latencies, the rate of FSK binding to the closed state of the channel must be very small. This rate, therefore, was approximated to be $0 / \mathrm{sec}$. The off rate of FSK from the closed channel, which has little effect on the amplitude or kinetics of the current, was estimated to be $10 / \mathrm{sec}$, in keeping with the recovery time-course data. The voltage dependence was assumed to be contained in the opening and closing transitions, not in the binding steps. The opening and closing rates of the channel with bound FSK (C*FSK $\leftrightarrow$
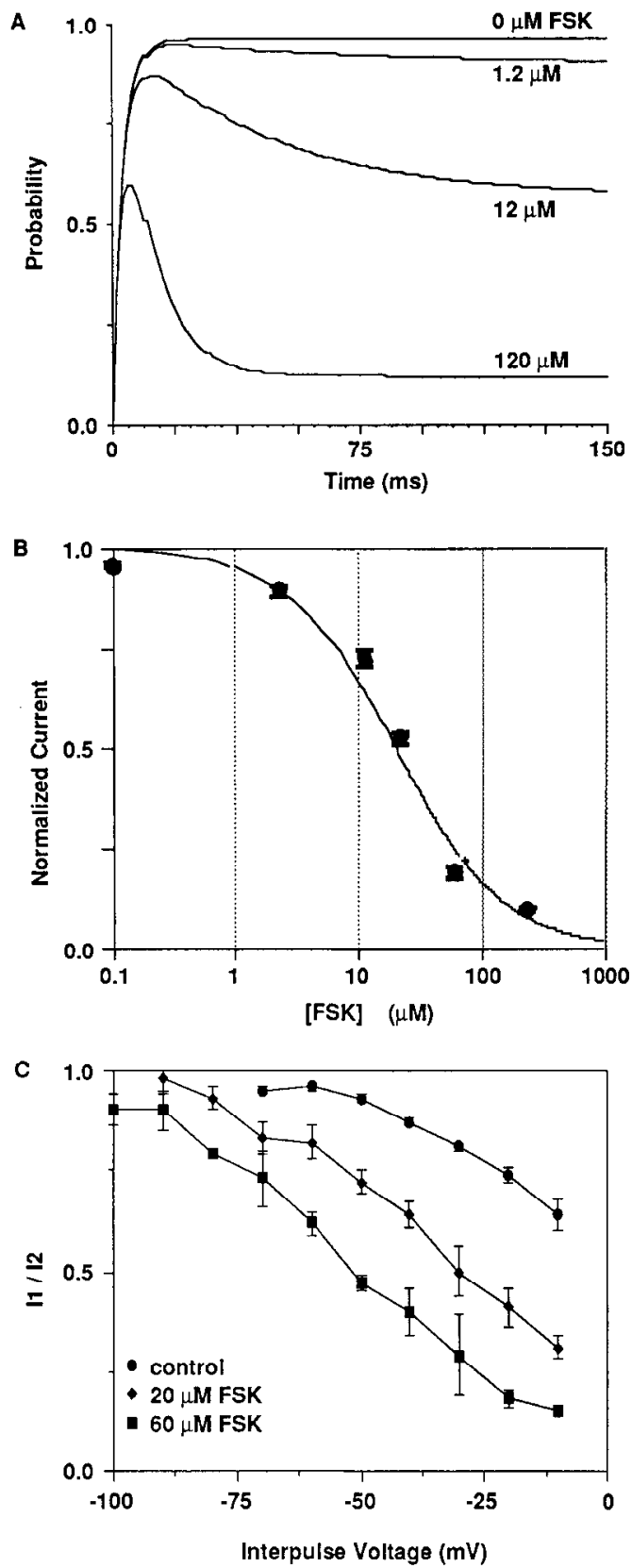

Figure 7. Predictions from Scheme 2 of the effect of increasing [FSK] on whole-cell $\mathrm{K}$ currents. $A$, Theoretical traces of whole-cell current in the presence of FSK were generated by Scheme 2, using the following parameters: $\alpha=250 / \mathrm{sec}, \beta=10 / \mathrm{sec}, k_{\text {on }}$ (FSK binding to the open state of the channel) $=106 / \mathrm{sec}, k_{\text {off }}$ (FSK unbinding from the closed channel) $=10 / \mathrm{sec}$, and the rate of FSK binding to the closed state of the channel $=0 / \mathrm{sec} . B$. Dependence of the peak current on increasing concentrations of [FSK]. Circles indicate averaged data from $\geq 3$ experiments (bars indicate SEM) in which the peak current was divided by the current at the end of the pulse. The solid line represents the prediction of Scheme 2, using parameters described above. $C$, Recovery from block. Each point is an average of $\geq 3$ experiments (bars indicate SEM). Circles represent control data; diamonds, $20 \mu \mathrm{M}$ FSK, and squares, $60 \mu \mathrm{M}$ FSK. Pulse protocol is similar to the 2-pulse protocol described in Figure $5 A$ except that the interpulse duration was constant at $1000 \mathrm{msec}$, and the interpulse voltage was varied from -100 to $-10 \mathrm{mV}$.

$\mathrm{O}$ FSK) were not determined in the current study. These rates are likely to be different from $\alpha$ and $\beta$. Figure $7 A$ shows predicted whole-cell currents in the presence of increasing concentrations of FSK derived using these parameters. These simulated cur- 
rents compare favorably to the effect of FSK shown in Figure 1. The concentration curve derived from these values (Fig. 7B) agrees well with the effect of increasing concentration of FSK on outward currents (Hoshi et al., 1988).

\section{FSK shifts voltage dependence of inactivation}

The application of lidocaine to voltage-dependent $\mathrm{Na}^{+}$channels results in a hyperpolarizing shift and decrease in slope of the inactivation curve (Hille, 1977; Hondeghem and Katzung, 1977; Bean et al., 1983). A similar shift was also observed with the application of FSK to $\mathrm{Kz}$ channels (Fig. $7 \mathrm{C}$ ). In this experiment, the ratio of the peak current was elicited using a 2-pulse protocol, while the interpulse voltage was varied. As the concentration of FSK was increased and more channels were blocked, the midpoint of inactivation shifted to the more negative direction with an accompanying decrease in slope.

\section{Discussion}

Our results show that FSK binds preferentially to the open state of the $\mathrm{Kz}$ channel. The effect of FSK on $\mathrm{Kz}$ channels can be adequately described by Scheme 2, in which FSK can bind and unbind from open and closed conformations of the $\mathrm{Kz}$ channel. In this scheme, an FSK-bound channel can move between open or closed conformations, and the presence of FSK does not change the voltage dependence of these transitions. The high affinity of FSK for the open state shifts the equilibrium toward the O*FSK state. Hyperpolarization drives the FSK-bound channel into the C*FSK state. The low affinity of FSK for the closed conformation of the channel results in the rapid unbinding of FSK from the channel. We have not directly addressed the effect of FSK on the inactivated states of the channels because the $\mathrm{Kz}$ channel does not inactivate within the time scale of these experiments. Scheme 2, however, can be extended to include the inactivated and FSK-bound inactivated conformations of the channel.

Preferential binding to the open state is also a characteristic of the binding of local anesthetics to the voltage-dependent $\mathrm{Na}^{+}$ channel. Hille (1977) developed the "modulated receptor hypothesis" to describe this interaction. In this hypothesis, the drug binds preferentially to the open conformation of the channel. For example, the block of $\mathrm{Na}^{+}$channels by charged anesthetics such as QX314 is characterized by (1) a use-dependent block that requires an open channel for the drug to bind, (2) a voltage-dependent block, suggesting that the drug moves into the pore, and (3) inability of the drug to unbind until the channel reopens (Frazier et al., 1970; Strichartz, 1973; Courtney, 1975). Hydrophobic or neutral compounds, such as lidocainc and benzocaine, differ in that these compounds can unbind from closed conformations of the channel (Khodorov et al., 1976; Hille, 1977; Schwarz et al., 1977).

Quaternary local-anesthetic molecules (e.g., QX-222 and QX314) also block AchRs (Steinbach, 1968; Adams, 1977; Neher and Steinbach, 1978). The blocking mechanism is similar to that described above for $\mathrm{Na}^{+}$channels in that the block is voltage dependent and open receptors are required for the quaternary local anesthetics to unbind (Neher and Steinbach, 1978). Quaternary local anesthetics act on the AchR only from the outside, unlike their action of $\mathrm{Na}^{+}$channels (Horn et al., 1980).

The interaction of FSK and the $\mathrm{Kz}$ channel is more similar to the block of the $\mathrm{Na}^{+}$channel by hydrophobic local anesthetics rather than that by charged local anesthetics. FSK binds preferentially to the open conformation of the channel. The lack of a rising phase in tail current measured in the presence of FSK indicates that FSK unbinds from the closed conformation. The hydrophobicity of FSK is important to its action on $\mathrm{Kz}$ channels as the more hydrophilic derivative, succinyl-FSK, is $\sim 10$-fold less effective in blocking outward $\mathrm{K}^{+}$currents in these cells (Hoshi et al., 1988). The effectiveness of FSK when applied to the outside, but not to the inside, of the $\mathrm{Kz}$ channel is reminiscent of the interaction between quaternary local anesthetics and $\mathrm{AChRs}$.

The effect of FSK, however, differs from the block of the voltage-dependent $\mathrm{Na}^{+}$channel or AchR by local anesthetics in that the interaction of FSK with the open state of the channel is not markedly voltage dependent. The apparent voltage dependence of the binding of FSK arises from the inherent voltage dependence of activation of $\mathrm{Kz}$ channcls. It is unlikely that FSK descreases ion flux through $\mathrm{Kz}$ channels by binding deeply within the pore where the effect of the electric field would be substantial.

An alternative mechanism to describe the effect of FSK on the $\mathrm{Kz}$ channel is that FSK binds to the channel in such a way as to alter only the rates of transition between conformational states of the channel. The effect would then be allosteric, in that FSK does not interact directly with the conduction pore of the channel, but perhaps binds at a hydrophobic region of the $\mathrm{Kz}$ channel protein or at a protein-lipid interface. Such a mechanism does not preclude block of the channel pore when FSK is bound to the channel (e.g., the binding of tetrodotoxin to batrachotoxin-activated $\mathrm{Na}^{+}$channels; Moczydlowski et al., 1984a,b; Green et al., 1987).

Because FSK interacts with the $\mathrm{Kz}$ channel differentially, depending on whether the channel is open or closed, a true dissociation constant for the drug cannot be accurately measured from macroscopic experiments such as those used by Hoshi et al. (1988). The true, overall, affinity of FSK for the $\mathrm{Kz}$ channel is a function of the voltage-dependent equilibria of both the open and closed conformations of the $\mathrm{Kz}$ channel. The FSK affinity is greater than suggested by the apparent dissociation constant for the open conformation and lower for the closed conformation, in a manner similar to the binding of local anesthetics to voltage-dependent $\mathrm{Na}^{+}$channels (Hille, 1977). Our results indicated that the dissociation constant is $\sim 20 \mu \mathrm{M}$ when the membrane is depolarized and the $\mathrm{Kz}$ channel is in the open conformation. However, when the membrane is hyperpolarized and the channel is closed, the dissociation constant is very large. Therefore, the direct effect of FSK will be observed primarily when the membrane potential is depolarized.

It is conceivable that FSK is an analogue for an endogenous compound that modulates the activity of the $\mathrm{Kz}$ or similar channel and thereby alters the shape of the action potential or firing frequency in some classes of cells. While the ringed structure of FSK is reminiscent of steroids, we have not yet been able to identify steroidal compounds with an effect similar to that of FSK on PC1 2 cells. Preliminary results suggest, however, that the calmodulin antagonist trifluorperizine is similarly effective.

Direct inhibitory effects of FSK have been reported with other membrane proteins, such as the glucose transporter, the acetylcholine receptor, and other $\mathrm{K}^{+}$currents. The classical effect of FSK on adenylate cyclase is distinct for these local-anestheticlike effects in that the 1,9-dideoxy-FSK derivative does not stimulate intracellular cAMP levels (Seamon et al., 1983). 1,9dideoxy-FSK, however, is as effective as FSK in interfering with 
ion and solute transport (McHugh and McGee, 1986; Hoshi et al., 1988; Krause et al., 1988; White, 1988; Harris, 1989). The hydrophobic derivative succinyl-FSK is also less effective (Lavis et al., 1987). The different affinities of FSK and derivatives for adenylate cyclase, glucose transporter, and ion channels suggest that the structure of the binding sites and the mechanisms of FSK block differ on these proteins.

\section{References}

Adams PR (1977) Voltage jump analysis of procaine action at frog end-plate. J Physiol (Lond) 268:291-318.

Bean BP, Cohen CJ, Tsien RW (1983) Lidocaine block of cardiac sodium channels. J Gen Physiol 81:613-642.

Coombs J, Thompson S (1987) Forskolin's effect on transient K current in nudibranch neurons is not reproduced by cAMP. J Neurosci 7:443-452.

Courtney KR (1975) Mechanism of frequency-dependent inhibition of sodium currents in frog myelinated nerve by the lidocaine derivative GEA. J Pharmacol Exp Ther 195:225-236.

Frazier DT, Narahashi T, Yamada M (1970) The site of action and active form of local anesthetics. II. Experiments with quaternary compounds. J Pharmacol Exp Ther 171:45-51.

Grassi F, Monaco L, Eusebi F (1987) Acetylcholine receptor channel properties in rat myotubes exposed to forskolin. Biochem Biophys Res Commun 147:1000-1007.

Green WN, Weiss LB, Andersen OS (1987) Batrachotoxin-modified sodium channels in planar lipid bilayers. Characterization of saxitoxin- and tetrodotoxin-induced channel closures. J Gen Physiol 89: 873-903.

Harris WR (1989) Forskolin reduces a transient potassium current in lobster neurons by a cAMP-independent mechanism. Brain Res 489: 59-66.

Hillc B (1977) Local ancsthetics: hydrophilic and hydrophobic pathways for the drug-receptor reaction. J Gen Physiol 69:497-515.

Hondeghem LM, Katzung BG (1977) Time- and voltage-dependent interactions of antiarrhythmic drugs with cardiac sodium channels. Biochim Biophys Acta 472:373-398.

Horn R, Brodwick MS, Dickey WD (1980) Asymmetry of the acetylcholine channel revealed by quaternary anesthetics. Science 210: 205-207.

Hoshi T, Aldrich RW (1988a) Voltage-dependent $\mathrm{K}^{+}$currents and underlying single $\mathrm{K}^{+}$channels in pheochromocytoma cells. J Gen Physiol 91:73-106.

Hoshi T, Aldrich RW (1988b) Gating kinetics of four classes of voltage-dependent $\mathrm{K}^{+}$channels in pheochromocytoma cells. J Gen Physiol 91:107-131.
Hoshi T, Garber SS, Aldrich RW (1988) Effect of forskolin on voltagegated $\mathrm{K}^{+}$channels is independent of adenylate cyclase activation. Science 240:1652-1655.

Kashiwagi A, Huecksteadt TP, Foley JE (1983) The regulation of glucose transport by cAMP stimulators via three different mechanisms in rat and human adipocytes. J Biol Chem 258:13685-13689.

Khodorov BI, Shishkova LD, Peganov E, Revenko S (1976) Inhibition of sodium currents in frog Ranvier node treated with local anesthetics. Biochim Biophys Acta 433:409-435.

Krause D, Lee SC, Deutsch C (1988) Forskolin effects on the voltagegated $\mathrm{K}^{+}$conductance of human T cells. Pflügers Arch 412:133-140.

Lavis VR, Lee DP, Shenolikar S (1987) Evidence that forskolin binds to the glucose transporter of human erythrocytes. J Biol Chem 262: 14571-14575.

McHugh EM, McGee RJ (1986) Direct anesthetic-like effects of forskolin on the nicotinic acetylcholine receptors of PC12 cells. J Biol Chem 261:3103-3106.

Moczydlowski E, Garber SS, Miller C (1984a) Batrachotoxin-activated $\mathrm{Na}^{+}$channels in planar lipid bilayers. Competition of tetrodotoxin block by $\mathrm{Na}^{+}$. J Gen Physiol 84:665-686.

Moczydlowski E, Hall S, Garber SS, Strichartz GS, Miller C (1984b) Voltage-dependent blockade of muscle $\mathrm{Na}^{+}$channels by guanidinium toxins. J Gen Physiol 84:687-704.

Neher E, Steinbach JH (1978) Local anaesthetics transiently block currents through single acetylcholine-receptor channels. J Physiol (Lond) 277:153-176.

Schwarz W, Palade PT, Hille B (1977) Local anesthetics. Effect of pH on use-dependent block of sodium channels in frog muscle. Biophys J 20:343-368.

Seamon KB, Daly JW, Metzger H, de Souza SN, Reden J (1983) Structure-activity relationships for activation of adenylate cyclase by the diterpene forskolin and its derivatives. J Med Chem 26:436-439.

Steinbach AB (1968) A kinetic model for the action of xylocaine on receptors for acetylcholine. J Gen Physiol 52:162-180.

Strichartz GR (1973) The inhibition of sodium currents in myelinated nerve by quaternary derivatives of lidocaine. J Gen Physiol 62:3757.

Wagoner PK, Pallotta BS (1988) Modulation of acetylcholine receptor desensitization by forskolin is independent of cAMP. Science 240 : 1655-1657.

Watanabe K, Gola M (1987) Forskolin interaction with voltage-dependent $\mathrm{K}$ channels in Helix is not mediated by cyclic nucleotides. Neurosci Lett 78:211-216.

White MM (1988) Forskolin alters acetylcholine receptor gating by a mechanism independent of adenylate cyclase activation. Mol Pharmacol 34:427-430. 